1 Hacettepe Journal of Mathematics and Statistics

Volume 46 (4) (2017), 559-565

\title{
A new class of Hardy spaces in the plane
}

\author{
Muhammed Ali Alan* and Nihat Gökhan Gögüş †
}

\begin{abstract}
We introduce new spaces that are extensions of the Hardy spaces and prove a removable singularity result for holomorphic functions within these spaces. Additionally we provide non-trivial examples.
\end{abstract}

Keywords: Hardy space, Removable singularity.

2000 AMS Classification: Primary 30H10, Secondary 30J99 AMS

Received : 01.08.2016 Accepted : 10.12.2016 Doi : 10.15672/HJMS.20174622759

\section{Introduction}

This paper deals with a construction of a holomorphic function space on an arbitrary open connected subset of the complex plane $\mathbb{C}$. In this paper we suggest a method of constructing a function space $W^{p}$ in any arbitrary domain. The definition of the norm on $W^{p}$ makes use of growth information of the function locally in the domain. We show that $W^{p}$ is Banach when $p \geq 1$ and prove a removable singularity theorem. This generalizes the result of M. Parreau in [8]. In the definition of $W^{p}$ we make use of the recently studied Poletsky-Stessin-Hardy (PSH) spaces. These spaces were introduced in several complex variables context in [9] and recently studied in planar domains in [1] and for the disk in the papers [10] and [11].

In general, PSH norm depends on the choice of the subharmonic exhaustion function which exists only when the domain is regular with respect to the classical Dirichlet problem. Our motivation for such a construction comes from the question that which subspaces of the classical Hardy space $H^{p}$ can be obtained as a Poletsky-Stessin-Hardy space. For example the subspace $z H^{p}$ of $H^{p}$ is not a Poletsky-Stessin-Hardy space because if the function $z$ belongs to this space, then so does the constant function 1 . However we show in section 4 that $B(z) H^{p}$ can be viewed as a $W^{p}$ space when $B$ is a finite Blaschke product.

*Syracuse University, Syracuse, NY 13244, USA, Email: malan@syr.edu

†Sabanci University, Tuzla , Istanbul 34956 TURKEY, Email: nggogus@sabanciuniv.edu

†Corresponding Author.

$\S$ This work was supported by a TUBITAK grant project $113 \mathrm{~F} 301$. 


\section{Poletsky-Stessin-Hardy spaces}

A function $u \leq 0$ on a bounded open set $G \subset \mathbb{C}$ is called an exhaustion on $G$ if the set

$$
B_{c, u}:=\{z \in G: u(z)<c\}
$$

is relatively compact in $G$ for any $c<0$. We denote the class of harmonic functions and subharmonic functions on a domain $\mathrm{G}$ by $\operatorname{har}(G)$ and $\operatorname{sh}(G)$, respectively. It is known that there is a subharmonic exhaustion function on $G$ if and only if $G$ is regular with respect to the classical Dirichlet problem. Let us denote the class of continuous subharmonic exhaustion functions on a domain $G$ by $\mathcal{E}(G)$. If $u$ is an exhaustion and $c<0$ is a number, we set

$$
u_{c}:=\max \{u, c\}, \quad S_{c, u}:=\{z \in G: u(z)=c\} .
$$

Since $u_{c}$ is a continuous subharmonic function the measure $\Delta u_{c}$ is well-defined. Following Demailly [2] we define

$$
\mu_{c, u}:=\Delta u_{c}-\chi_{G \backslash B_{c, u}} \Delta u
$$

where $\chi_{\omega}$ is the characteristic function of a set $\omega \subset G$. We shall call these measures as Demailly measures.

If $u$ is a negative subharmonic exhaustion function on $G$, then the Demailly-LelongJensen formula takes the form

$$
\int_{S_{c, u}} v d \mu_{c, u}=\int_{B_{c, u}}(v \Delta u-u \Delta v)+c \int_{B_{c, u}} \Delta v
$$

where $\mu_{c, u}$ is the Demailly measure which is supported in the level sets $S_{c, u}$ of $u$ and $v \in \operatorname{sh}(G)$. This formula is the one variable version of the result which was proved by Demailly [2]. Let us recall that by [2] if $\int_{G} \Delta u<\infty$, then the measures $\mu_{c, u}$ converge as $c \rightarrow 0$ weak-* to a measure $\mu_{u}$ supported in the boundary $\partial G$.

Let $u \in \operatorname{sh}(G)$ be an exhaustion function which is continuous with values in $\mathbb{R} \cup\{-\infty\}$. Following [9] we set

$$
s h_{u}(G):=s h_{u}:=\left\{v \in \operatorname{sh}(G): v \geq 0, \sup _{c<0} \int_{S_{c, u}} v d \mu_{c, u}<\infty\right\},
$$

and

$$
H_{u}^{p}(G):=H_{u}^{p}:=\left\{f \in \operatorname{hol}(G):|f|^{p} \in s h_{u}\right\}
$$

for every $p>0$. We write

$$
\|v\|_{u}:=\sup _{c<0} \int_{S_{c, u}} v d \mu_{c, u}=\int_{G}(v \Delta u-u \Delta v)
$$

for the norm of a nonnegative function $v \in \operatorname{sh}(G)$ and set

$$
\|f\|_{u, p}:=\sup _{c<0}\left(\int_{S_{c, u}}|f|^{p} d \mu_{c, u}\right)^{1 / p}
$$

for the norm of a holomorphic function $f$ on $G$. Let us write $\|f\|_{u}$ when $p=1$. It is known in view of $\left[9\right.$, Theorem 4.1] that $H_{u}^{p}$ is a Banach space when $p \geq 1$. It is clear that the function 1 belongs to $H_{u}^{p}$ if and only if the Demailly measure $\mu_{u}$ has finite mass. If $G$ is a regular bounded domain in $\mathbb{C}$ and $w \in G$, then we have the Green function $v(z)=g_{G}(z, w)$ which is a subharmonic exhaustion function for $G$.

The following result is obtained in [1]. 
2.1. Theorem. Let $G$ be a bounded domain and $u \in \mathcal{E}(G)$. Let $p>0$. The following statements are equivalent:

i. $f \in H_{u}^{p}(G)$.

ii. There exists a least harmonic function $h$ in $G$ which belongs to the class $s h_{u}$ so that $|f|^{p} \leq h$ on $G$. Furthermore,

$$
\|f\|_{u, p}^{p}=\int_{G} h \Delta u=\|h\|_{u} .
$$

Now let $G$ be a bounded domain with $C^{1}$ boundary or a bounded simply connected domain with rectifiable boundary. Let $u \in \mathcal{E}(G)$ and $p \geq 1(p>0$ if $G$ is simply connected). Then the space $H_{u}^{p}(G)$ (thinking of boundary values) is a closed subspace of the weighted space $L^{p}\left(V_{u} d \sigma\right)$ on the boundary $\partial G$, where (see [1]) $d \sigma$ is the usual Lebesgue measure on $\partial G$ and

$$
V_{u}(\zeta)=\int_{G} P_{G}(z, \zeta) \Delta u(z), \quad \zeta \in \partial G
$$

is the balayage of the positive measure $\Delta u$ to the boundary $\partial G$. Then $V_{u}(\zeta)=\frac{\partial u}{\partial \mathbf{n}}(\zeta)$ is the directional derivative of $u$ in the normal direction at a point $\zeta \in \partial G$ (see [4] and [11]). The next results are restatements from [9] and they establish basic observations on the classes of Hardy spaces.

2.2. Proposition. [9, Corollary 3.2] Let $v$ be a continuous subharmonic exhaustion function on a bounded regular domain $G$ and let $v(z)=g(z, w)$ be the Green function. Then $\operatorname{sh}_{u}^{p}(G) \subset s h_{v}^{p}(G)$ and there is a constant $c$ such that $\|\varphi\|_{v} \leq c\|\varphi\|_{u}$ for every nonnegative subharmonic function $\varphi$ on $G$.

2.3. Proposition. [9, Corollary 3.2] Let $u$ and $v$ be continuous subharmonic exhaustion functions on $G$ and let $K$ be a compact set in $G$ such that $b v(z) \leq u(z)$ for some constant $b>0$ and all $z \in G \backslash K$. Then $s h_{v} \subset s h_{u}$ and $\|\varphi\|_{u} \leq b\|\varphi\|_{v}$ for every $\varphi \in s h_{v}$.

The following result is basically contained in the proof of [9, Theorem 3.6] taking $n=1$.

2.4. Proposition. Let $v$ be a continuous subharmonic exhaustion function on $G, K \subset G$ be compact and $V \subset \subset G$ be an open set containing $K$. Suppose that there exists a constant $s>0$ so that $v(z) \leq s g_{G}(z, w)$ for every $w \in K$ and $z \in G \backslash \bar{V}$. Then

$$
\varphi(w) \leq \frac{s}{2 \pi}\|\varphi\|_{v}, \quad w \in K
$$

for every nonnegative $\varphi \in \operatorname{sh}(G)$.

\section{Hardy spaces in arbitrary open sets}

In this section we propose a way to define weighted Hardy spaces in arbitrary planar domains. For Hardy spaces in multiply connected domains we refer to [3]. Let us set the notation first. Let $\Omega$ be a domain, $E \subset \Omega$ be a compact polar subset and let $\Omega_{j}$ be a sequence of regular domains so that $\Omega_{j} \subset \Omega$ and the union of all $\Omega_{j}$ is the open set $\Omega \backslash E$. Also for each $j$ let $u_{j} \in \mathcal{E}\left(\Omega_{j}\right)$, that is, $u_{j}$ is a subharmonic exhaustion function for $\Omega_{j}$. We define the class $W^{p}$ of holomorphic functions on $\Omega$ as follows:

$$
W^{p}:=\left\{f \in \operatorname{hol}(\Omega): \sup _{j}\|f\|_{u_{j}, p}<\infty\right\} .
$$

Let us define

$$
\|f\|_{W^{p}}:=\sup _{j}\|f\|_{u_{j}, p}
$$


for any $f \in W^{p}$. We will write $W^{p}\left[u_{j}, \Omega, E\right]$ if we wish to emphasize the sequence of functions $u_{j}$ used in the definition or the underlying domain $\Omega$ and the polar set $E$. Before showing that $\left(W^{p},\|\|_{W^{p}}\right)$ is a Banach space we need the following removable singularity theorem for bounded holomorphic functions due to Lelong.

3.1. Theorem. [7, p.35], [5, p. 107] Let $E$ be a relatively closed pluri-polar set and let $f$ be holomorphic in $\Omega \backslash E$. Suppose that $f$ is bounded on $\Omega \backslash E$. Then $f$ has a unique holomorphic extension to the whole of $\Omega$.

We prove an auxiliary result.

3.2. Theorem. Let $f_{n}$ be a holomorphic function on a domain $\Omega$ and $E$ be a compact polar set in $\Omega$. Suppose that $f_{n}$ converges uniformly to a function $f$ on compact subsets of $\Omega \backslash E$. Then the function $f$ can be extended to a holomorphic function on $\Omega$.

Proof. Let $\Gamma$ be a bounded open region in $\Omega$ with piecewise smooth boundary $\gamma$ so that $E \subset \Gamma \subset \bar{\Gamma} \subset \Omega$. Since $\left|f_{n}\right|$ converges uniformly to $|f|$ on $\gamma$, we see that $\sup _{n}\left|f_{n}\right|$ is uniformly bounded on $\gamma$, that is, there exists a number $M$ so that $\left|f_{n}\right| \leq M$ on $\gamma$ for every $n$. We write $P_{\Gamma} \varphi$ for the Poisson integral of a continuous function $\varphi$ on $\gamma$. Then

$$
\left|f_{n}(z)\right| \leq P_{\Gamma}\left|f_{n}\right|(z) \leq M
$$

for every $n$ for every $z \in \Gamma$. Therefore $|f(z)| \leq M$ for every $z \in \Gamma \backslash E$. By Theorem $3.1 f$ has a holomorphic extension to $\Gamma$. Since $f$ is already holomorphic outside of $\Gamma$ we conclude that $f$ can be extended to a holomorphic function on $\Omega$.

We can now prove that $\left(W^{p},\|\|_{W^{p}}\right)$ is Banach.

3.3. Theorem. $\left(W^{p},\|\|_{W^{p}}\right)$ is a Banach space for $p \geq 1$.

Proof. If $\|f\|_{W^{p}}=0$, then $\|f\|_{u_{j}, p}=0$, that is why $f=0$ in $\Omega_{j}$ for every $j$. Hence $f=0$ on $\Omega \backslash E$, and since $E$ is polar, $f=0$ on $\Omega$. The other properties of norm can be easily checked for $\|f\|_{W^{p}}$. So let us prove that it is complete. Take a Cauchy sequence $\left\{f_{n}\right\}$ from $W^{p}$. This implies first that the sequence of holomorphic functions $\left\{f_{n}\right\}$ is Cauchy in $H_{u_{j}}^{p}$ for every $j$. We conclude that $f_{n}$ converges uniformly to a function $f$ on every compact subset of $\Omega_{j}$ for each $j$, hence on every compact subset of $\Omega \backslash E$. By Theorem $3.2 f$ extends to a holomorphic function to the whole of $\Omega$.

To prove that $f \in W^{p}$ we will now show that $\left\|f_{n}-f\right\|_{W^{p}}$ converges to zero. Given $\varepsilon>0$ there exists an integer $N \geq 1$ so that

$$
\sup _{j}\left\|f_{n}-f_{m}\right\|_{u_{j}, p}<\varepsilon
$$

whenever $n, m \geq N$. This gives that

$$
\left\|f_{n}-f\right\|_{W^{p}}=\sup _{j}\left\|f_{n}-f\right\|_{u_{j}, p} \leq \varepsilon
$$

for every $n \geq N$. Therefore $\left\|f_{n}-f\right\|_{W^{p}}$ converges to zero and $f \in W^{p}$.

It is known that a polar set is a removable singularity for the classical Hardy spaces in the plane (see [6] and [8]). The next result can be considered as a removable singularity theorem for the $W^{p}$ spaces. There by $\left.W^{p}\left[u_{j}, \Omega, E\right]\right|_{\Omega \backslash E}$ we denote the class of restrictions of the functions from $W^{p}\left[u_{j}, \Omega, E\right]$ to $\Omega \backslash E$.

3.4. Theorem. Let $\bar{\Omega}_{j} \subset \Omega_{j+1}, E \subset \Omega$ be a compact polar set for every $j$ and let $p>0$. If there exists an open set $U \subset \Omega \backslash E$ so that $\sup _{j} u_{j}(z) \leq \ell<0$ for every $z \in U$, then

$$
\left.W^{p}\left[u_{j}, \Omega, E\right]\right|_{\Omega \backslash E}=W^{p}\left[u_{j}, \Omega \backslash E, \emptyset\right] .
$$


Proof. The inclusion $\left.W^{p}\left[u_{j}, \Omega, E\right]\right|_{\Omega \backslash E} \subset W^{p}\left[u_{j}, \Omega \backslash E, \emptyset\right]$ is immediate from the definitions. To prove the reverse inclusion we claim that if $f$ belongs to the space $W^{p}\left[u_{j}, \Omega \backslash E, \emptyset\right]$, then $f$ extends to a holomorphic function to the whole set $\Omega$. Since $f \in H_{u_{j}}^{p}\left(\Omega_{j}\right)$, according to Theorem 2.1 we see that the function $h_{j}:=P_{\Omega_{j}}\left(|f|^{p}\right)$ has the properties that $h_{j} \in \operatorname{har} \cap s h_{u_{j}}\left(\Omega_{j}\right)$ and that $|f|^{p} \leq h_{j}$ on $\Omega_{j}$. Then $h_{j} \leq h_{j+1}$, and thanks to the Harnack theorem the limit $h=\lim h_{j}$ is a harmonic function on $\Omega \backslash E$ unless $h=\infty$ identically everywhere. Let $z_{0} \in U$ and $r>0$ so that $\left\{z:\left|z-z_{0}\right| \leq r\right\} \subset U$. We claim that $h\left(z_{0}\right)<\infty$. There exists a constant $s>0$ so that $\ell<s g_{\Omega}\left(z, z_{0}\right) \leq s g_{\Omega_{j}}\left(z, z_{0}\right)$ for every $z \in \Omega_{j}$ with $\left|z-z_{0}\right|=r, j \geq 1$. Harmonicity of the Green's function $g_{\Omega_{j}}\left(z, z_{0}\right)$ on $\Omega_{j} \backslash\left\{z:\left|z-z_{0}\right| \leq r\right\}$ implies that $u_{j}(z) \leq \ell<s g_{\Omega_{j}}\left(z, z_{0}\right)$ for every $z \in \Omega_{j} \backslash\left\{z:\left|z-z_{0}\right| \leq r\right\}$ for every $j \geq 1$. By Theorem 2.1 and Proposition 2.4 we see that

$$
\frac{2 \pi}{s} h_{j}\left(z_{0}\right) \leq\left\|h_{j}\right\|_{p, u_{j}}=\|f\|_{p, u_{j}} \leq\|f\|_{W^{p}\left[u_{j}, \Omega \backslash E, \emptyset\right]}<\infty
$$

for every $j \geq 1$. This proves that $h\left(z_{0}\right)<\infty$. Hence $h \in \operatorname{har}(\Omega \backslash E)$ and satisfies $|f|^{p} \leq h$. Now this means $f$ belongs to the Hardy class of functions mentioned in [8]. By [8, Theorem A] $f$ admits a unique holomorphic extension to $\Omega$ and therefore $f \in W^{p}\left[u_{j}, \Omega, E\right]$. This completes the proof.

\section{Examples}

In view of [9, Proposition 3.5] $H_{u}^{p} \subset H^{p}$ when $u$ is a subharmonic exhaustion on the disk. It is our purpose to construct examples of subsets of the classical Hardy space $H^{p}$ on the disk which can be described using the Hardy spaces of the form $W^{p}$. The next examples are of this sort. In the next example we construct a family of exhaustion functions inside the unit disk to descripe the space of functions in $H^{p}$ which are zero at 0 .

4.1. Example. For any $0<R<1$ let $\Gamma_{R}$ denote the annulus

$$
\Gamma_{R}:=\{z \in \mathbb{C}: R<|z|<1\} .
$$

If $t>0$, define a subharmonic exhaustion function $u_{t}$ in $\Gamma_{R}$ by

$$
u(z):=u_{t}(z):=u_{t, R}(z):=\max \left\{t \log \left(\frac{R}{|z|}\right), \log |z|\right\} .
$$

Some properties of $u_{t}$ are listed below.

(1) $u_{t}(z)=0$, if $|z|=1$ or $|z|=R$.

(2) We solve $t \log \left(\frac{R}{|z|}\right)=\log |z|$ to get $|z|=R^{t / t+1}$ and hence

$$
u_{t}(z)= \begin{cases}t \log \left(\frac{R}{|z|}\right) & \text { if } R<|z| \leq R^{t / t+1} \\ \log |z|, & \text { if } R^{t / t+1}<|z|<1\end{cases}
$$

(3) We compute the measure $\mu_{u}$ of $u$.

$V_{u}\left(e^{i \theta}\right)=\left.\frac{\partial u}{\partial \mathbf{n}}\right|_{z=e^{i \theta}}=1$

and

$V_{u}\left(R e^{i \theta}\right)=\left.\frac{\partial u}{\partial \mathbf{n}}\right|_{z=R e^{i \theta}}=t / R$

for every $\theta \in[0,2 \pi]$. Hence, for any positive measurable function $\varphi$ on $\partial \Gamma_{R}$ we have

$\int_{\partial \Gamma_{R}} \varphi d \mu_{u}=\frac{t}{2 \pi R} \int_{0}^{2 \pi} \varphi\left(R e^{i \theta}\right) d \theta+\frac{1}{2 \pi} \int_{0}^{2 \pi} \varphi\left(e^{i \theta}\right) d \theta$. 
Now we are ready to state the main purpose of this example.

4.2. Theorem. Let $H^{p}$ be the classical Hardy space in the unit disc for $p>0$ and $k \geq 1$ be an integer. Let $\left(R_{n}\right)$ be any sequence of numbers converging to 0 so that $0<R_{n}<1$. Take $\alpha$ so that $1-k p \leq \alpha<1-k p+p$. Then we have

$$
z^{k} H^{p}=W^{p}\left[u_{R_{n}^{\alpha}, R_{n}}, \mathbb{D},\{0\}\right]
$$

and two spaces have equivalent norms.

Proof. Let $W^{p}=W^{p}\left[u_{R_{n}^{\alpha}, R_{n}}, \mathbb{D},\{0\}\right]$. If $h \in z^{k} H^{p}$, we will show that $h \in W^{p}$. Let $h=z^{k} f$, where $f \in H^{p}$. Then

$$
\begin{aligned}
\left\|z^{k} f\right\|_{W^{p}}^{p} & =\sup _{n}\left\|z^{k} f\right\|_{u_{R_{n}^{\alpha}, p}}^{p} \\
& =\sup _{n}\left(\frac{R_{n}^{\alpha+k p-1}}{2 \pi} \int_{0}^{2 \pi}\left|f\left(R_{n} e^{i \theta}\right)\right|^{p} d \theta+\frac{1}{2 \pi} \int_{0}^{2 \pi}\left|f\left(e^{i \theta}\right)\right|^{p} d \theta\right) \\
& \leq \sup _{n}\left(R_{n}^{\alpha+k p-1}+1\right)\|f\|_{H^{p}}^{p} \leq 2\|f\|_{H^{p}}^{p}<\infty .
\end{aligned}
$$

Hence $h \in W^{p}$ and $z^{k} H^{p} \subset W^{p}$.

Conversely, let $h \in W^{p}$. By the definition of the norm of $W^{p}$ (by item (3) above for instance) it is clear that $\|h\|_{H^{p}} \leq\|h\|_{W^{p}}$ for every $h \in W^{p}$, that is, $W^{p} \subset H^{p}$. We will show that $h \in z^{k} H^{p}$. Suppose on the contrary that $h(z)=z^{m} f(z)$, where $0 \leq m \leq k-1$, $f \in H^{p}$ and $|f(0)|>0$. Then

$$
\begin{aligned}
\|h\|_{W^{p}} & \geq \sup _{n} \frac{R_{n}^{\alpha+(k-1) p-1}}{2 \pi} \int_{0}^{2 \pi}\left|f\left(R_{n} e^{i \theta}\right)\right|^{p} d \theta \\
& \geq \sup _{n} R_{n}^{\alpha+(k-1) p-1}|f(0)|^{p}=\infty .
\end{aligned}
$$

The contradiction shows that $h(z)=z^{k} f(z)$ for some $f \in H^{p}$. Hence $W^{p}=z^{k} H^{p}$.

Finally we can do the previous construction for finite Blaschke products.

4.3. Theorem. Let $a_{1}, \cdots, a_{N}$ be distinct points in $\mathbb{D}$ and let

$$
B(z):=\prod_{j=1}^{N}\left(\frac{z-a_{j}}{1-\bar{a}_{j} z}\right)^{k_{j}},
$$

where $k_{j} \geq 1$ are integers. Let $p>0$. Then there exists a sequence $\left\{\Omega_{n}\right\} \subset \mathbb{D}$ of $N+1$-connected domains and functions $u_{n} \in \mathcal{E}\left(\Omega_{n}\right)$ so that

$$
B(z) H^{p}=W^{p}\left[u_{n}, \mathbb{D}\right]
$$

and two spaces have equivalent norms.

Proof. Choose $R>0$ small enough so that the circles

$$
C_{j}=\left\{z:\left|\frac{z-a_{j}}{1-\bar{a}_{j} z}\right|^{k_{j}}=R\right\},
$$

$j=1, \cdots, N$, are pairwise disjoint. Let $\Omega_{R}$ be the $N+1$-connected domain with boundary $\partial \mathbb{D} \cup \cup_{j=1}^{N} C_{j}$. For each $j$ choose $\alpha_{j}$ so that $-k_{j} p \leq \alpha_{j}<-k_{j} p+p$. Let $\psi_{R}$ be the function defined on $\partial \mathbb{D}$ by 1 , on $C_{j}$ by $R^{\alpha}$. Then $\psi_{R}$ is lower semicontinuous on $\Omega_{R}, \psi_{R} \geq t_{R}>0$ for some constant $t=t_{R}$ and by [4, Theorem 2.1] there exists a subharmonic exhaustion $u_{R} \in \mathcal{E}\left(\Omega_{R}\right)$ so that $\partial u_{R} / \partial \mathbf{n}=\psi_{R}$ on $\partial \Omega_{R}$. 
Now let $0<R_{n}<R$ be numbers decreasing to 0 and consider the space $W^{p}=$ $W^{p}\left[u_{R_{n}}, \mathbb{D}\right]$. If $h=B f \in B(z) H^{p}$, then

$$
\begin{aligned}
\|B f\|_{W^{p}}^{p} & =\sup _{n}\left(\sum_{j=1}^{N} R_{n}^{\alpha_{j}+k_{j} p} \int_{C_{j}}|f(\zeta)|^{p} d \sigma_{j}+\frac{1}{2 \pi} \int_{0}^{2 \pi}\left|f\left(e^{i \theta}\right)\right|^{p} d \theta\right) \\
& \leq \sup _{n}\left(\sum_{j=1}^{N} R_{n}^{\alpha_{j}+k_{j} p}+1\right)\|f\|_{H^{p}}^{p} \leq(N+1)\|f\|_{H^{p}}^{p}<\infty .
\end{aligned}
$$

Hence $h \in W^{p}$ and $B H^{p} \subset W^{p}$.

Conversely, let $h \in W^{p}$. By the definition of the norm of $W^{p}$ it is clear that $\|h\|_{H^{p}} \leq$ $\|h\|_{W^{p}}$ for every $h \in W^{p}$, that is, $W^{p} \subset H^{p}$. We will show that $h \in B H^{p}$. Suppose on the contrary that the multiplicity $m_{j}$ of zero of $h(z)$ at $a_{j}$ is strictly less than $k_{j}$, that is $0 \leq m_{j} \leq k_{j}-1$ for some $j$. Then $h(z)=\left(\frac{z-a_{j}}{1-\bar{a}_{j} z}\right)^{m_{j}} f(z)$ for a function $f \in H^{p}$ with $\left|f\left(a_{j}\right)\right|>0$ and therefore,

$$
\begin{aligned}
\|h\|_{W^{p}} & \geq \sup _{n} R_{n}^{\alpha_{j}+\left(k_{j}-1\right) p} \int_{C_{j}}|f(\zeta)|^{p} d \sigma_{j} \\
& \geq C \sup _{n} R_{n}^{\alpha_{j}+\left(k_{j}-1\right) p}\left|f\left(a_{j}\right)\right|^{p}=\infty .
\end{aligned}
$$

The contradiction shows that $h(z)=B(z) f(z)$ for some $f \in H^{p}$. Hence $W^{p}=B(z) H^{p}$.

\section{References}

[1] Alan, M. A. and Göğüş, N. G. Poletsky-Stessin-Hardy spaces in the plane, Complex Anal. Oper. Theory 8 (2014), no. 5, 975-990.

[2] Demailly, J. P. Mesure de Monge-Ampère et mesures plurisousharmonique, Math. Z. 194 (1987), 519-564.

[3] Duren, P. L. Theory of $H^{p}$ spaces, Pure and Applied Mathematics, Vol. 38 Academic Press, New York-London 1970.

[4] Göğüş, N. G. Structure of weighted Hardy spaces in the plane, Filomat 30, (2016) no. 2, 473-482.

[5] Jarnicki, P. and Pflug, P. Extension of Holomorphic Functions, De Gruyter Expositions in Mathematics 34

[6] Järvi, P. Removable singularities for $H^{p}$-functions, Proc. Amer. Math. Soc. 86 (1982), 596598.

[7] Lelong, P. Fonctions plurisousharmoniques et formes differentielles positives, Gordon and Breach, New York, 1968.

[8] Parreau, M. Sur les moyennes des fonctions harmoniques et analytiques et la classification des surfaces de Riemann, Ann. Inst. Fourier (Grenoble) 3 (1951), 103-197.

[9] Poletsky, E. A. and Stessin, M. I. Hardy and Bergman spaces on hyperconvex domains and their composition operators, Indiana Univ. Math. J. 57 (2008), no. 5, 2153-2201.

[10] Şahin, S., Poletsky-Stessin Hardy spaces on domains bounded by an analytic Jordan curve in $\mathbb{C}$, Complex Var. Elliptic Equ. 60 (2015), no. 8, 1114-1132

[11] Shrestha, K. R. Weighted Hardy spaces on the unit disk, Complex Anal. Oper. Theory 9 (2015), no. 6, 1377-1389. 
